\title{
Inhalt des acht und zwanzigsten Bandes.
}

$$
\text { E r s t e s } H \text { e f } t \text {. }
$$

1. Einige Beınerkungen aus der alten, mittelalterlichen und neueren Baukunst; zur Auffindung von Verbesserungen. Von dem Herrn Oberbaumeister Engelhardt zu Cassel in Hessen. . . . . . . . . . . . . . . . . Seite 1

2. Übersicht der Geschichte der Baukunst, mit Rüchsicht auf die allgemeine Culturgeschichte. Von Herrn Regierungs - und Baurath C. A. Rosenthal zu Magdeburg. (Fortseizung der Ábhandilung No. 2., 6 . und 8. im 13ten, No. 1., 7., 8. und 12. im 14ten, No. 1., 9., 11. und 15. im 15ten, No. 10. im 16ten, No. 3., 5. und 10. im 17ten, No. 4. im 18ten, No. 2. im 20ten, No. 9. im 22ten, No. 1., 9. und 13. im 25ten, No. 2. und 12. im 26ten, No. 3. und 10. im 27ten Bande.)

$$
Z \text { w e i } t \text { e s } H \text { e f } t \text {. }
$$

3. Der Tunnel von Lioran. (Aus den ${ }_{n}$ Annales des ponts et chaussées. Jahrgang 1846. 2ter Band." Von Herrn Ruclle, Brücken - und WegeIngenieur zu Aurillac.) . . . . . . . . . . . . . . . . . .

4. Übersicht der Geschichte der Baukunst, mit Rücksicht auf die allgemeine Culturgeschichte. Von Herrn Regierungs - und Baurath C. A. Rosenthal zu Magdeburg. (Fortsetzung der Abhandlung No. 2., 6. und 8. im 13ten, No. 1., 7., 8. und 12. im 14ten, No. 1., 9., 11. und 15. im 15ten, No. 10. im 16 ten, No. 3., 5. und 10. im 17ten, No. 4. im 18ten, No. 2. im 20ten, No. 9. im 22ten, No. 1., 9. und 13. im 25ten, No. 2. und 12. im 26ten, No. 3. und 10. im 27ten und No. 2. in diesem Bande.) . . . . . . . . . . . . -126

5. Technische Mittel zur Erhaltung des Credits jetziger und künftiger EisenbahnActien. Von dem Herrn Oberbaumeister Engelhard zu Cassel in Hessen.

6. Über die Veränderungen der Erd-Oberfläche durch das Wasser. Von dem Herrn Ingenieur He/s zu Götlingen. 


\section{D r i t e s $H$ ef $t$.}

7. Übersicht der Geschichle der Baukunst, mit Rücksicht auf die allgemeine Culturgeschichte. Von Herrn Regierungs- und Baurath C. A. Rosenthal zu Magdeburg. (Schlufs der Abhandlung No. 2., 6. und S. im 13ten, No. 1., 7., 8. und 12. im 14ten, No. 1., 9., 11. und 15. im 15ten, No.10. im 16ten, No. 3., 5. und 10. im 17ten, No. 4. im 18ten, No. 2. im 20ten, No. 9. im 22ten, No. 1., 9. und 13. im 25ten, No. 2. und 12. im 26ten, No. 3. und 10. im 27ten, No. 2. und 4. in diesem Bande.) . . . . . . . . . . . Seite 177

$\rtimes$. Über das Princip des kleinsten Widerstandes. Von dem Herrn Bau-Conducteur Schefflev zu Braunschweig. . . . . . . . . . . . . . . - 199

9. Über dic Beziehung zwischen dem Alter und dem Werth der Gebäude. Von Herrn Dr. E. Segritz zu Eldena. . . . . . . . . . . . .

10. Der Tunnel von Lioran. (Aus den ,Annales des ponts et chaussées Jahrgang 1846. 2ter Band.") Von Herrn Ruelle, Brücken - und WegeIngenieur zu Aurillac. (Fortselzung der Abhandlung No. 3. im vorigen Hefte.)

$$
V \text { i e r } t \text { e s } H \text { e f } t
$$

11. Der Tunnel von Lioran. (Aus den ${ }$ Annales des ponts et chaussées. Jahrgang 1846. 2ter Band.”) Von Herrn Ruelle, Brücken - und WegeIngenieur zu Aurillac. (Schlufs der Abhandlung No. 3. und No. 10. in diesem Bande.) . . . . . . . . . . . . . . . . . . . .

12. Beitrag zum Wegebau: ein Vorschlag, die Kosten möglichst zu vermindern und der Verwüstung des Bedeckungsmaterials vorzubeugen. Vom Herrn Dr. theol. F. H. Germar zu Heide in Norderdithmarschen. . . . . . - 304

13. Von den Gesetzen der Bewegung der Luft (der Aërodynamik). Nach D'Aubuisson de Voisins. . . . . . . . . . . . . . . . . .

14. Nachtrag zur , Übersicht der Geschichte der Baukunst S. 46 im 20ten Bande d. Journ." Von Herrn Regierungs - und Baurath C. A. Rosenthal zu Mag-

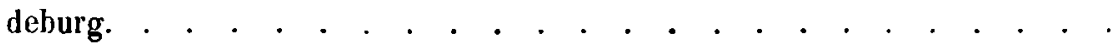

15. Ausführliches Inhalts-Verzeichnifs der „Übersicht der Geschichte der Baukunst" des Herrn Regierungs - und Bauraths Rosenthal zu Magdeburg. Von Demselben. 


\title{
J o u r n a l
}

\author{
f ü $\mathbf{r}$
}

\section{d i e B a uk u n s t. \\ I n z w a n g l o s e n H e f $t$ e $n$.}

Herausgegeben

vo n

\section{Dr. A. L. Crelle,}

Käniglich - Preufsischem Geheimen - Ober-Baurathe, Mitgliede der Königlichen Akademie der Wissenschaflen zu Berlin, Correspondenten der Kaiserlichen Akademie der Wissenschaften zu St. Petersburg und der Königlichen Akademieen der Wissenschaften zu Neapel und Brüssel, auswärtigem Mitgliede der Königlichen Akademie der Wissenschaftel zu Stockholm, Ehrenmilgliede der Hamburger Gesellschaft zur Verbreitung der mathematischen Wissenschaften.

\section{Acht und zwanzigster Band.}

Erstes Heft.

Mit einer Figurenitafel.

B e $r \mid$ i n.

B e $\mathrm{i}$ G. R e i ne $r$.

1849 . 
